\title{
ASPECTS OF THE THEORY OF DERIVATIONS
}

\author{
GERARD J. MURPHY \\ Department of Mathematics, University College \\ Cork, Ireland
}

\begin{abstract}
We survey some old and new results in the theory of derivations on Banach algebras. Although our overview is broad ranging, our principal interest is in recent results concerning conditions on a derivation implying that its range is contained in the radical of the algebra.

In this paper we present a survey of some aspects of the theory of derivations on Banach algebras. No attempt is made at completeness; rather, our intention is to cover the basic theory and to discuss some recent results. We are principally interested in certain results generalising the Singer-Wermer theorem; more precisely, in certain generalisations involving conditions on a derivation that ensure that its range is contained in the radical of the algebra. We also discuss other results, and some problems, related to this generalised Singer-Wermer theory.

A very important aspect of the theory of derivations centres on the question of innerness of all the derivations on a given Banach algebra. We give a brief overview of this theory and of the closely related results pertaining to the problem of whether all the derivations of an algebra can be made "inner" in some bigger algebra.

We shall occasionally include (easier) proofs to give some indication of the methods used in this area. Also, we shall give details of some examples where it is easy to do so.

We shall be concerned, for the most part, in this exposition only with bounded everywhere-defined derivations on Banach algebras, with only occasional references to unbounded derivations. There exists a well-developed theory of denselydefined derivations that has a wide variety of applications. The interested reader is referred to Sakai's recent book [19] for details. This book also contains an extensive bibliography.
\end{abstract}

1991 Mathematics Subject Classification: 46H99, 46J99, 47B47.

The paper is in final form and no version of it will be published elsewhere. 
We begin our survey, in the following section, by recalling some basic facts and early results concerning derivations.

1. Elements of the theory of derivations. Let $A$ be a Banach algebra. A derivation on $A$ is a linear operator $d: A \rightarrow A$ such that $d(a b)=a d b+(d a) b$ for all elements $a, b \in A$. If $b$ is a fixed element of $A$, then the operator $d$ on $A$ defined by $d(a)=[a, b]=a b-b a$ is a bounded derivation on $A$, called an inner derivation. Not all derivations are inner and one of the interesting problems of the theory is to find conditions on an algebra which ensure that it has inner derivations only. (We discuss some results and open problems concerning innerness in Section 4.)

If $d$ is a derivation on $A$, the condition $d(a b)=a d b+(d a) b$ is, of course, an analogue of the Leibniz rule for ordinary differentiation and, as with the usual rule, it can be easily generalised, to give

$$
d^{n}(a b)=\sum_{j=0}^{n}\left(\begin{array}{l}
n \\
j
\end{array}\right)\left(d^{j} a\right) d^{n-j} b .
$$

This is called the Leibniz formula and will be used frequently below.

Another useful and easy observation: If $I$ is an ideal of $A$, then so is $d(I)+I$ (the proof is trivial). In many cases, one can get a much stronger result. For instance, if $I$ is a closed ideal of a $\mathrm{C}^{*}$-algebra $A$, then $d(I) \subseteq I$. For, $I$ is the linear span of its positive elements and each such element is of the form $a^{2}$ for some $a \in I$. Hence, $d\left(a^{2}\right)=a d a+(d a) a$ and therefore $d\left(a^{2}\right)$ belongs to $I$. Consequently, $d(I) \subseteq I$, as claimed. We shall discuss in more detail below the question of invariance under derivations of certain closed ideals in arbitrary Banach algebras.

One of the earliest results (1955) in the theory of derivations is the celebrated theorem of I. M. Singer and J. Wermer [22] which asserts that every bounded derivation on a commutative Banach algebra has range contained in the radical - thus, for semisimple commutative Banach algebras, there are no non-zero derivations. We shall discuss this result in more detail in Section 2 and present a proof there (as we shall see, it is little more than an exercise in the use of the generalised Leibniz formula).

The statement of the Singer-Wermer theorem specifies that the derivation in question has to be bounded. However, it was immediately conjectured by Singer and Wermer that their result would still hold if the boundedness condition were dropped. Since, as we observed above, the proof of the Singer-Wermer theorem is very easy, it might be supposed that the unbounded case is a little harder, but not very much so. In fact, however, it is a lot harder! The conjecture of Singer and Wermer was confirmed only in 1988, by M. P. Thomas [23]; that is, he showed that every derivation (bounded or unbounded) on a commutative Banach algebra has range contained in the radical. The proof is long and very non-trivial.

In light of the preceding remarks, two questions spring to mind, namely, do unbounded derivations actually exist and, if they do, do there exist, neverthe- 
less, large classes of Banach algebras on which all derivations are automatically bounded. The answer to both questions is affirmative. An early result of S. Sakai (1960) asserts that all derivations on $\mathrm{C}^{*}$-algebras are bounded (for a proof, see $[13$, p. 328]). Sakai's result was extended by B. E. Johnson and A. M. Sinclair [6], who showed that all derivations on semisimple Banach algebras are automatically bounded.

However, as is well known, unbounded derivations do exist in general. We present here an example of an unbounded derivation on a commutative Banach algebra. Our example has the merit that the radical of the algebra involved is as small as it can be if the algebra is to admit unbounded derivations - to be precise, the radical is one-dimensional (by the Johnson-Sinclair result, if the radical were zero, all derivations on the algebra would be bounded).

1.1. ExAmple. Let $A$ be the (commutative) Banach algebra which is the completion of the algebra of all sums

$$
\lambda r+\sum_{j=1}^{n} \lambda_{j} e_{j}
$$

where $\lambda, \lambda_{1}, \ldots, \lambda_{n}$ are arbitrary scalars, $e_{1}, \ldots, e_{n}, \ldots$ are fixed mutually orthogonal idempotents, and $r$ is a fixed element such that $r^{2}=0$ and $e_{j} r=r e_{j}=0$ for all $j$. The norm is given by

$$
\left\|\lambda r+\sum_{j=1}^{n} \lambda_{j} e_{j}\right\|=\max \left\{\left(\sum_{j=1}^{n}\left|\lambda_{j}\right|^{2}\right)^{1 / 2},\left|\lambda-\sum_{j=1}^{n} \lambda_{j}\right|\right\} .
$$

It is a result of $\mathrm{W}$. Bade, $\mathrm{P}$. Curtis and C. Feldman that $A$ has $\operatorname{radical} \operatorname{rad}(A)$ equal to $\mathbb{C} r$ and that there exists a non-closed ideal $I$ of $A$ such that $A=I \oplus \mathbb{C} r$ (see [1]). Let $d$ be the derivation on $A$ defined by setting $d(a+\lambda r)=\lambda r$, where $a \in I$ and $\lambda \in \mathbb{C}$. Then $\operatorname{ker}(d)=I$, so $d$ cannot be bounded, as $I$ is not closed in $A$.

We now discuss a result on invariance of primitive ideals under bounded derivations which is interesting in its own right and a very useful tool in the theory of derivations (an application is given in Section 3). The result is due to Sinclair [21] and since it is not difficult, we give its proof (incidentally, one should compare this proof with that of Theorem 2.1 below which is essentially the usual proof of the Kleinecke-Shirokov theorem).

1.2. Theorem. Let $d$ be a bounded derivation on a Banach algebra $A$ and let $I$ be a primitive ideal of $A$. Then $d(I) \subseteq I$.

P r o of. Let $a$ be an element of $I$. It is easily seen by application of the Leibniz formula that $d^{j}\left(a^{k}\right) \in I$ for all $0 \leq j<k$. Hence, by induction, $d^{n}\left(a^{n}\right)-n !(d a)^{n} \in$ $I$ for $n>0$. To see this, let us assume the result for $n=k$ and show it for $n=k+1$ : 
We have

$$
\begin{aligned}
d^{k+1}\left(a^{k+1}\right) & =d d^{k}\left(a^{k} a\right) \\
& =\sum_{j=0}^{k}\left(\begin{array}{c}
k \\
j
\end{array}\right)\left[d^{j+1}\left(a^{k}\right) d^{k-j}(a)+d^{j}\left(a^{k}\right) d^{k+1-j}(a)\right] \\
& =k d^{k}\left(a^{k}\right) d a+d^{k}\left(a^{k}\right) d a+\text { terms belonging to } I \\
& =(k+1) !(d a)^{k+1}+\text { terms belonging to } I
\end{aligned}
$$

It follows that in the Banach algebra $A / I$,

$$
\left\|(d a+I)^{n}\right\|=\left\|d^{n}\left(a^{n}\right)+I\right\| / n ! \leq(\|d\|\|a\|)^{n} / n ! .
$$

Taking $n$th roots of both sides and letting $n \rightarrow \infty$, we see that the spectral radius of $d a+I$ is zero, so $d a+I$ is quasinilpotent in $A / I$. Since $d(I)+I$ is an ideal of $A$, therefore $(d(I)+I) / I$ is an ideal of $A / I$ consisting of quasinilpotent elements. Hence, $(d(I)+I) / I$ is contained in $\operatorname{rad}(A / I)[15$, p. 57]. Since $I$ is assumed to be primitive, $\operatorname{rad}(A / I)=0$, so $d(I)+I=I$; that is, $d(I) \subseteq I$.

The obvious question arising from the preceding theorem is whether boundedness of $d$ can be dropped in the hypothesis. The answer is not known - that is, it is unknown whether an unbounded derivation on a Banach algebra leaves all primitive ideals invariant. However, some partial results have recently been obtained in the general situation. For instance, if a primitive ideal of a Banach algebra $A$ is infinite-codimensional in $A$, then it is invariant under all derivations of $A$. Also, if $d$ is an unbounded derivation on $A$, then the set of primitive ideals of $A$ not invariant for $d$ is finite (but we do not know whether it is empty). For an account of these results, which are the accomplishment of a number of mathematicians, see [24].

2. The Singer-Wermer theorem and related results. In this section we discuss some generalisations of the Singer-Wermer theorem and the relationship of this theorem to certain other results, in particular, to the well known theorem of D. C. Kleinecke and F. V. Shirokov.

Let $d$ be a bounded derivation on a Banach algebra $A$. It was observed by I. Kaplansky [7] that we may always regard $d$ as "inner" in a bigger algebra. More precisely, there is a Banach algebra $B$ containing $A$ as a closed subalgebra and there exists an element $b \in B$ such that $d(a)=[a, b]$ for all $a \in A$. To see this, note first that if $A$ is non-unital, then we may extend $d$ to a bounded derivation on the unitisation of $A$ by setting $d(1)=0$. Thus, we may assume that $A$ is unital. Now take $B$ to be the Banach algebra of all bounded linear operators on $A$ and embed $A$ isometrically in $B$ via the left regular representation. We can now take $b=-d \in B$ to complete the proof.

This trick of realising $d$ as inner in a bigger algebra is useful in some contexts. For example, we can use it to see that the Kleinecke-Shirokov theorem implies the Singer-Wermer theorem. First, recall that the former theorem asserts that if 
$a$ and $b$ are elements of a Banach algebra such that $[a,[a, b]]=0\left({ }^{1}\right)$, then $[a, b]$ is quasinilpotent $[8,20]$. To see how one can deduce the Singer-Wermer theorem from this, let $d$ be a bounded derivation on a Banach algebra $A$ and suppose that $a$ is an element of $A$ such that $[a, d a]=0$. Choose a Banach algebra $B$ containing $A$ as above such that there is an element $b \in B$ for which $d(c)=[c, b]$ for all $c \in A$. Then $[a,[a, b]]=0$, so by the Kleinecke-Shirokov theorem, $[a, b]=d a$ is quasinilpotent. As the radical is just the set of quasinilpotent elements in the commutative case, this result clearly implies the Singer-Wermer theorem and can be thought of as a "local" version of it. (For more along these lines, see [10].)

It does not appear to be known whether the condition $[a, d a]=0$, for a given element $a$ of $A$, implies that $d a$ is quasinilpotent in the case that $d$ is unbounded. However, it has recently been shown by M. Mathieu and V. Runde [11] and by M. Brešar and J. Vukman [3] that if $[a, d a]=0$ for all $a \in A$, then the range of $d$ is contained in the radical of the algebra (with no assumption of boundedness of $d$ ).

We complete our discussion of this circle of ideas by proving a result on bounded derivations that gives both the Kleinecke-Shirokov and Singer-Wermer theorems as immediate consequences.

2.1. TheOrem. Let $d$ be a bounded derivation on a Banach algebra $A$ and suppose that $a$ is an element of $A$ such that $d^{2}(a)=0$. Then da is quasinilpotent.

Proof. Simple inductions using the Leibniz formula repeatedly show that the condition $d^{2} a=0$ implies that $d^{n+1}\left(a^{n}\right)=0$ and hence $d^{n}\left(a^{n}\right)=n !(d a)^{n}$ for all positive integers $n$. Since $\left\|d^{n}\left(a^{n}\right)\right\| \leq\|d\|^{n}\|a\|^{n}$, therefore $\left\|(d a)^{n}\right\|^{1 / n} \leq$ $(n !)^{-1 / n}\|d\|\|a\|$, so $d a$ has spectral radius equal to zero.

A special case of Theorem 2.1 is that in which $d$ is the inner derivation defined by $d b=[a, b](b \in A)$. The result then shows that if $[a,[a, b]]=0$, then $[a, b]$ is quasinilpotent; that is, the Kleinecke-Shirokov theorem is a special case of Theorem 2.1. Hence, the Singer-Wermer theorem follows from Theorem 2.1 also, since, as we observed above, it is a consequence of the Kleinecke-Shirokov theorem. The proof of Theorem 2.1 given above is essentially just a slight adaptation of the proof of the Kleinecke-Shirokov theorem. Alternatively, if one prefers, one may easily deduce Theorem 2.1 from the Kleinecke-Shirokov theorem by using Kaplansky's trick.

If one drops the assumption that $d$ is bounded in the hypothesis of Theorem 2.1, the result - that $d a$ is quasinilpotent-remains true. This was shown recently by Thomas [24]. The proof is not easy.

3. Derivations mapping into the radical. We showed in the preceding section that if $d$ is a bounded derivation on a Banach algebra $A$ such that $[a, d a]=0$ for all $a \in A$, then $d a$ is quasinilpotent for all $a \in A$. In fact, more can be said,

$\left({ }^{1}\right)$ Editorial note: See also condition $(*)$ in the paper of V. S. Shul'man in this volume, where further consequences are discussed. 
for if a derivation on $A$ (bounded or unbounded) has range contained in the set of quasinilpotent elements, then the range actually lies in the radical. This is a recent result due to Yu. V. Turovskil and V. S. Shul'man [25]. The proof of the bounded case given in [10] illustrates nicely some of the techniques used in this context, so we present it here.

3.1. TheOrem. Let $d$ be a bounded derivation on a Banach algebra $A$ and suppose that the range of $d$ is contained in the set of quasinilpotent elements of A. Then $d(A) \subseteq \operatorname{rad}(A)$.

Proof. Without loss of generality we may suppose that $A$ is unital. If $I$ is a primitive ideal of $A$, then $d(I) \subseteq I$, by Theorem 1.2. Therefore, there is an induced bounded derivation $d^{\prime}$ on the quotient Banach algebra $A / I$ defined by $d^{\prime}(a+I)=d a+I$ and clearly $d^{\prime}(a+I)$ is quasinilpotent for all $a \in A$. If the theorem is true in the case of primitive Banach algebras, we can therefore deduce that $d^{\prime}(A / I) \subseteq \operatorname{rad}(A / I)=0$, so $d(A) \subseteq I$. It then follows that $d(A) \subseteq \operatorname{rad}(A)$, since the radical is the intersection of all the primitive ideals. Thus, to prove the theorem, we may suppose that $A$ is primitive. We may therefore regard $A$ as acting irreducibly as an algebra of bounded operators on a Banach space $X$.

If $x \in X, a \in A$ and $a(x)=0$, we claim that $d a(x)=0$. For if not, then irreducibility of $A$ implies that there exists an element $b$ of $A$ such that $b d a(x)=x$. Hence, $d(b a)(x)=b d a(x)+(d b) a(x)=x$, so 1 is an eigenvalue of $d(b a)$, contradicting the fact that $d(b a)$ is quasinilpotent.

We now claim that for any $x \in X$ and $a \in A$, the vectors $a(x)$ and $d a(x)$ are linearly dependent. For if not, then by the Jacobson density theorem, there exists an element $b \in A$ such that $b a(x)=0$ and $b d a(x)=-a(x)$. By the preceding paragraph, $d(b a)(x)=0$ and therefore $(d b) a(x)=(d(b a)-b d a)(x)=a(x)$, so 1 is an eigenvalue of $d b$, a contradiction.

If now $a$ is an invertible element of $A$, then by what we have shown, there exists for each element $x$ of $X$ a scalar $\lambda(x)$ such that $d a(x)=\lambda(x) a(x)$. This implies that $a^{-1} d a=\lambda$, for some scalar $\lambda$. However, because $d a$ is not invertible and $a$ is, it follows that $\lambda=0$. Thus, we have shown that $d a=0$ for all invertible elements $a$ of $A$, from which it is immediate that $d=0$.

Note that the preceding theorem extends an earlier result of Pták [14], who proved the theorem in the case of inner derivations.

We mention also a recent result of $\mathrm{M}$. Brešar [2] that also belongs to this circle of ideas. Denoting by $r(\cdot)$ the spectral radius, he shows that if $d$ is an inner derivation of a unital Banach algebra $A$ such that there is a positive number $M$ for which $r(d a) \leq \operatorname{Mr}(a)$ for all $a \in A$, then the range of $d$ is contained in the radical of $A$. Brešar conjectures that the result holds for arbitrary bounded derivations $\left({ }^{2}\right)$.

$\left({ }^{2}\right)$ Editorial note: See also Conjecture 3 in the paper of M. Mathieu in this volume, and the editorial note at the end of that paper. 
J. Zemánek has also posed an interesting question relating to this context, namely, if $d$ is a derivation of a unital Banach algebra $A$ such that $\sup _{a} r\left(a^{-1} d a\right)$ is finite, where $a$ varies over the invertible elements of $A$, is the range of $d$ necessarily contained in the radical. He shows that the answer is affirmative in the case that $d$ is an inner derivation $[26]\left(^{3}\right)$.

4. Conditions implying innerness of derivations. In this section we consider the question whether all derivations on a given algebra $A$ are inner, or can be made "inner" in a bigger algebra. We shall confine ourselves here to $\mathrm{C}^{*}$-algebras, where a deep and extensive theory has been developed.

Suppose now that $A$ is a $\mathrm{C}^{*}$-algebra. We consider first the question whether every derivation of $A$ is inner. There are two important cases in which the answer is affirmative: firstly, when $A$ is a $\mathrm{W}^{*}$-algebra (a $\mathrm{W}^{*}$-algebra can be characterised as a $\mathrm{C}^{*}$-algebra which is isometrically linearly isomorphic to the dual space of a Banach space) and secondly, when $A$ is a unital simple $\mathrm{C}^{*}$-algebra (a simple $\mathrm{C}^{*}$-algebra is one having no non-zero proper closed ideals). Both results are due to Sakai $[16,17,18]$.

However, it is not true, in general, that all derivations on a $\mathrm{C}^{*}$-algebra are inner and it is easy to give counter-examples. For instance, let $H$ be an infinitedimensional Hilbert space and let $B(H)$ and $K(H)$ denote the $\mathrm{C}^{*}$-algebras of all bounded and compact operators on $H$, respectively. Choose any operator $u \in B(H)$ not belonging to $K(H)+\mathbb{C} 1$. Then it is easily verified that the derivation $d$ on $K(H)$ defined by setting $d v=[v, u]$ for all $v \in K(H)$ is not inner. Note also that $K(H)$ is simple (but not unital). However, it is the case that for any derivation $d$ on $K(H)$, there exists an element $u$ of $B(H)$ such that $d v=[v, u]$ for all $v \in K(H)$. This is a special case of another result of Sakai [18] which asserts that if $A$ is a non-unital simple $\mathrm{C}^{*}$-algebra and $M(A)$ is its multiplier algebra, then for each derivation $d$ of $A$, there exists an element $b$ of $M(A)$ such that $d a=[a, b]$ for all $a \in A$. (For the definition and elementary properties of the multiplier algebra, see $[12,13]$. In the case $A=K(H)$, we have $M(A)=B(H)$.) For other results on "innerness" in the multiplier algebra, see [13, Section 8.6].

It is useful, at this point, to slightly extend the definition of a derivation. Let $A$ be a $C^{*}$-subalgebra of a $C^{*}$-algebra $B$. If $d$ is a bounded linear map from $A$ to $B$ such that $d\left(a_{1} a_{2}\right)=a_{1} d a_{2}+\left(d a_{1}\right) a_{2}$ for all $a_{1}, a_{2} \in A$, we call $d$ a derivation. By the Gelfand-Naimark representation theorem, each $\mathrm{C}^{*}$-algebra $A$ can be regarded as a $\mathrm{C}^{*}$-subalgebra of $B(H)$ for some Hilbert space $H$, so it is natural to pose the question whether every derivation $d: A \rightarrow B(H)$ is inner; that is, does there exist an element $b \in B(H)$ such that $d a=[a, b]$ for all $a \in A$. The answer to this question is not known, even for the case that $A$ is a von Neumann algebra on $H$ (though the answer is known, and affirmative, for certain large classes of von Neumann algebras). There is a very nice result due to E. Christensen [4]

$\left({ }^{3}\right)$ Editorial note: Matej Brešar verified the general case on October 4, 1993. 
which gives a reformulation of this problem in terms of complete boundedness. To define the latter concept, we need to recall firstly that for each $\mathrm{C}^{*}$-algebra $A$, the algebra $M_{n}(A)$ of all $n \times n$ matrices with entries in $A$ can be endowed with a unique norm making it a $\mathrm{C}^{*}$-algebra, and secondly that each linear map $\varphi$ from $A$ to a $\mathrm{C}^{*}$-algebra $B$ has an inflation $\varphi_{n}$ from $M_{n}(A)$ to $M_{n}(B)$ defined by setting $\varphi_{n}\left(a_{i j}\right)=\left(\varphi\left(a_{i j}\right)\right)$. We say that $\varphi$ is completely bounded if all the inflations $\varphi_{n}$ are bounded and $\sup _{n}\left\|\varphi_{n}\right\|<\infty$. Christensen's reformulation is the following result.

4.1. Theorem. If $A$ is a $C^{*}$-subalgebra of $B(H)$, then a derivation $d: A \rightarrow$ $B(H)$ is inner if and only if $d$ is completely bounded.

This result therefore gives a connection with the flourishing area of completely bounded maps. For nice expositions of this result and related matters and of the theory of completely bounded maps, see [5, 9].

\section{References}

[1] W. Bade and P. Curtis, Homomorphisms of commutative Banach algebras, Amer. J. Math. 82 (1960), 589-608.

[2] M. Brešar, Derivations decreasing the spectral radius, Arch. Math. (Basel) 61 (1993), 160-162.

[3] M. Brešar and J. Vukman, On left derivations and related mappings, Proc. Amer. Math. Soc. 110 (1990), 7-16.

[4] E. Christensen, Extensions of derivations II, Math. Scand. 50 (1882), 111-122.

[5] E. Christensen and A. M. Sinclair, A survey of completely bounded operators, Bull. London Math. Soc. 21 (1989), 417-448.

[6] B. E. Johnson and A. M. Sinclair, Continuity of derivations and a problem of Kaplansky, Amer. J. Math. 90 (1968), 1067-1073.

[7] I. Kaplansky, Functional analysis, in: Some Aspects of Analysis and Probability, Surveys Appl. Math. 4, New York, 1958, 1-34.

[8] D. C. Kleinecke, On operator commutators, Proc. Amer. Math. Soc. 8 (1957), 535-536.

[9] M. Mathieu, Derivations and completely bounded maps on $C^{*}$-algebras. A survey, preprint, University of Tübingen, 1990.

[10] M. Mathieu and G. J. Murphy, Derivations mapping into the radical, Arch. Math. (Basel) 57 (1991), 469-474.

[11] M. Mathieu and V. Runde, Derivations mapping into the radical, II, Bull. London Math. Soc. 24 (1992), 485-487.

[12] G. J. Murphy, $C^{*}$-Algebras and Operator Theory, Academic Press, New York, 1990.

[13] G. K. Pedersen, $C^{*}$-Algebras and their Automorphism Groups, Academic Press, New York, 1979.

[14] V. Pták, Commutators in Banach algebras, Proc. Edinburgh Math. Soc. 22 (1979), 207211.

[15] C. E. Rickart, General Theory of Banach Algebras, Van Nostrand, New York, 1960.

[16] S. Sakai, Derivations of $W^{*}$-algebras, Ann. of Math. 83 (1966), 273-279.

[17] - Derivations of simple $C^{*}$-algebras, J. Funct. Anal. 2 (1968), 202-206.

[18] - Derivations of simple $C^{*}$-algebras II, Bull. Soc. Math. France 99 (1971), 259-263. 
[19] S. Sakai, Operator Algebras in Dynamical Systems, Cambridge University Press, Cambridge, 1991.

[20] F. V. Shirokov, Proof of a conjecture of Kaplansky, Uspekhi Mat. Nauk 11 (4) (1956), 167-168 (in Russian).

[21] A. M. Sinclair, Continuous derivations on Banach algebras, Proc. Amer. Math. Soc. 20 (1969), 166-170.

[22] I. M. Singer and J. Wermer, Derivations on commutative normed algebras, Math. Ann. 129 (1955), 260-264.

[23] M. P. Thomas, The image of a derivation is contained in the radical, Ann. of Math. 128 (1988), 435-460.

[24] -, Primitive ideals and derivations on non-commutative Banach algebras, Pacific J. Math. 159 (1993), 139-152.

[25] Yu. V. Turovskiŭ and V. S. Shul'man, Conditions for massiveness of the range of the derivation of a Banach algebra and associated differential operators, Math. Notes 42 (1987), 669-674.

[26] J. Zemánek, Properties of the spectral radius in Banach algebras, in: Spectral Theory, Banach Center Publ. 8, PWN, Warszawa, 1982, 579-595. 